\title{
Learning-set de reversões de discriminações simples em macaco-prego*
}

\author{
Romariz da Silva Barros**, Carlos Rafael Fernandes Picanço, \\ Thiago Dias Costa \& Carlos Barbosa Alves de Souza \\ Universidade Federal do Pará, Belém, Brasil
}

\begin{abstract}
RESUMO
A aquisição repetida de discriminações simples pode determinar uma crescente eficiência na aprendizagem dessas discriminações. Esse efeito é conhecido como learning-set. $\mathrm{O}$ mesmo efeito pode ser observado em reversões repetidas de uma mesma discriminação ou em reversões repetidas de discriminações simples combinadas (RRDSC). O presente estudo relata a aplicação de RRDSC com até seis estímulos (três positivos e três negativos), simultaneamente, com um macaco-prego (Cebus cf. apella). Ao longo das repetidas reversões, avaliou-se o efeito de learning-set em duas variáveis: a) número de acertos processados até o critério de seis acertos consecutivos e b) número de tentativas até o critério. Os dados indicam a obtenção de learning-set nas RRDSC. Discutem-se os procedimentos que mais contribuíram para essa demonstração.
\end{abstract}

Palavras-chave: reversões repetidas de discriminações simples combinadas; learning-set; Cebus cf apella.

\begin{abstract}
Learning-set of simple discrimination reversals in a capuchin monkey

Repeated acquisition of simple discrimination may determine an increasing efficiency in learning such discriminations. This effect is known as learning-set. The same effect can be observed on repeated reversals of the same discrimination or on repeated yoked reversals of simple discriminations (RYRSD). The present study reports the use of RYRSD with up to six stimuli (three positive and three negative) simultaneously with a capuchin monkey (Cebus cf apella). Over the course of the repeated reversals, the learning-set effect was evaluated taking into account two variables: a) number of correct responses processed until criterion of six consecutive correct responses is reached and b) number of trials until the criterion is reached. The data indicate learning-set in simple discrimination on yoked reversals. We discuss the procedures that contributed the most to this demonstration.
\end{abstract}

Keywords: repeated reversals of yoked simple discriminations; learning-set; Cebus cf apella.

A exposição a múltiplos exemplares de um mesmo tipo de treino discriminativo tem como efeito a aquisição cada vez mais rápida das discriminações, o que é conhecido como learning-set (Harlow, 1949). Na medida em que o número de exemplares da tarefa apresentados ao sujeito aumenta, o número de tentativas necessárias para o aprendizado diminui, o que pode culminar em desempenhos perfeitos ou quase perfeitos após poucas tentativas.
O efeito de learning-set pode ser também observado em reversões repetidas de uma mesma discriminação ou em reversões repetidas de discriminações simples combinadas (RRDSC). O procedimento de RRDSC consiste em treinar um conjunto de discriminações simples (por exemplo, A1+/A2-; B1+/B2-; C1+/C2-) e, uma vez aprendidas as discriminações, processar reversões sucessivas das funções de todos os estímulos ao mesmo tempo (por exemplo, A2+/A1-; B2+/B1-; $\mathrm{C} 2+/ \mathrm{C} 1-)$.

\footnotetext{
Nota dos autores: Pesquisa financiada pelo CNPq, Bolsa de Produtividade para o primeiro e o último autor e apoio à pesquisa (Edital Universal 480912/2011-4) e INCT-ECCE.

** Endereço para correspondência: Romariz da Silva Barros - romarizsb@gmail.com.
} 
O efeito de learning-set, em sujeitos não humanos, tem sido relatado raramente e só é obtido em estudos de longa duração (ou seja, estudos que incluem centenas de sessões experimentais). A longa duração dos estudos pode ser inclusive uma dificuldade em se tratando de pesquisas com ratos, por exemplo. A este respeito, Costa, Barros, Galvão e Reis (2007) relataram um estudo cujo objetivo foi explorar um procedimento que permitisse a redução da duração de pesquisas sobre reversões de discriminações em não humanos.

Estudos com reversões repetidas de discriminações combinadas em geral têm foco na investigação da formação de classes funcionais (Kastak, Schusterman \& Kastak, 2001; Vaughan, 1988). Vaughan considerou que encontrou evidência de formação de classes funcionais de estímulos quando verificou que o contato dos sujeitos com as contingências revertidas no início de uma sessão determinava a reversão do desempenho para os estímulos restantes na sessão (reversões espontâneas). De acordo com Vaughan, esse desempenho era função do processo de partição de um conjunto em subconjuntos, ou seja, da aprendizagem do responder de forma generalizada para os estímulos de um subconjunto e discriminadamente entre estímulos de subconjuntos diferentes.

De acordo com Lionello-DeNolf, Canovas, Souza, Barros e McIlvane (2008), a formação de classes funcionais pode ser um desdobramento lógico de um continuum de efeitos do learning-set de reversões de discriminações. Desse modo, se um determinado procedimento não produz evidências de learning-set de reversões, ele possivelmente não levará à formação de classes funcionais de estímulos.

Em outras palavras, o efeito de learning-set não é suficiente para explicar reversões exibidas prontamente sem erros com alguns estímulos quando a reversão foi diretamente treinada apenas com alguns outros estímulos do mesmo conjunto (ou seja, formação de classes funcionais). Entretanto, a obtenção de learning-set pode predizer o potencial de um determinado procedimento de reversões de discriminações simples para produzir a formação de classes funcionais. Dessa maneira, o refinamento metodológico dos procedimentos de reversões repetidas de discriminações simples, com potencial obtenção de learning-set, pode impulsionar o estudo da formação de classes funcionais em sujeitos não humanos e em humanos com desenvolvimento severamente atrasado.
Costa (2008) relatou um estudo com macacosprego (Cebus cf. apella) no qual foi documentado não apenas learning-set, mas também fortes evidências de formação de classes funcionais. O procedimento consistia no treino de reversões repetidas de discriminações simples combinadas com a apresentação de seis estímulos (caixas de madeira - três com função positiva [S+] e três com função negativa [S-]) em um aparato tridimensional. Era requerido que os sujeitos se deslocassem até os estímulos e então os escolhessem.

Inicialmente as discriminações e reversões eram treinadas com dois, quatro e finalmente com seis estímulos. Em seguida uma reversão era treinada com quatro estímulos somente (dois $\mathrm{S}+$ e dois $\mathrm{S}$-). Tão logo o critério de aprendizagem fosse atingido (seis tentativas corretas consecutivas), os dois estímulos não apresentados neste treino de reversão eram reinseridos já com as funções revertidas. A primeira resposta a esse par de estímulos era crítica para a avaliação da formação de classes. A história de reforçamento mais recente com esse par de estímulos determinaria que os sujeitos respondessem ao $\mathrm{S}$ - (que funcionou como S+ na última vez que foi apresentado aos sujeitos). A reversão da função dos outros estímulos, contudo, poderia determinar a reversão não treinada da função dos dois estímulos reintroduzidos, caso houvesse formação de classes funcionais. Os dados mostraram fortes evidências de formação de classes, ou seja, os sujeitos, desde a primeira resposta ao par de estímulos reintroduzidos, respondiam aos mesmos já com as funções revertidas.

Esse estudo, quando comparado com outros estudos da literatura sobre a formação de classes funcionais (Dube, Callahan \& Mcllvane, 1993; Schusterman, Kastak \& Schusterman, 2001; Vaughan, 1988) apresenta as seguintes características inovadoras: (1) os estímulos eram caixas de madeira apresentadas em um aparato tridimensional trazido até a gaiola-viveiro dos sujeitos, de maneira que os mesmos tinham que se deslocar até os estímulos e procurar por comida em seu interior, aumentando o custo para erros e aproveitando o repertório natural de busca de alimento bem desenvolvido nos macacos-prego; (2) todos os estímulos S+ e S- eram apresentados simultaneamente, podendo ser apresentados até seis estímulos ao mesmo tempo no aparato; (3) respostas exclusivamente aos $\mathrm{S}+$ produziam acesso a comida extra, o que reforça um padrão de busca de alimento mais eficiente no aparato. Nos estudos anteriores, os estímulos eram 
apresentados em painéis de resposta que se ajustavam pouco às características da espécie, com baixo custo para erros e com as discriminações apresentadas sucessivamente e não simultaneamente.

A apresentação dos estímulos (caixas de madeira) e o registro das respostas (procurar por comida em seu interior) eram feitos manualmente no estudo de Costa (2008), dadas as características artesanais do aparato. O presente estudo apresenta uma versão automatizada bidimensional do mesmo procedimento de Costa (2008). O objetivo foi avaliar as alterações de procedimento introduzidas nas reversões de discriminações simples no sentido de obter um procedimento automatizado (como os demais estudos da literatura) mas possivelmente com potencial para produzir efeito de learning-set de reversões de discriminações simples combinadas mostrado no estudo de Costa (2008). O presente estudo, portanto, pretende acrescentar contribuições para o avanço na metodologia de estudos subsequentes sobre a formação de classes funcionais de estímulos.

\section{MÉTODO}

Sujeito: Participou do estudo Bongo (M16), um macaco prego (Cebus cf. apella) macho, adulto (aproximadamente 13 anos no início do procedimento), com história experimental envolvendo discriminações simples e condicionais com até quatro estímulos apresentados simultaneamente. Bongo vivia em uma gaiola $(2,50 \times 2,50 \times 2,50 \mathrm{~m})$ juntamente com outros macacos da mesma espécie, com livre acesso a água. A alimentação era fornecida uma vez ao dia. Nenhum esquema adicional de privação foi utilizado. As condições de vida em cativeiro, dieta, cuidados veterinários, e os procedimentos de coleta foram aprovados pelo Comitê de Ética em Pesquisa Animal do Instituto de Ciências Biológicas da Universidade Federal do Pará (licença \# CEPAE PS001/2005-UFPA), em acordo com as normas locais e internacionais sobre o tratamento e manipulação de animais para fins de pesquisa. O biotério onde ficavam alojados os animais é um criadouro de animais silvestres para fins científicos, registrado junto ao IBAMA (número 207419, código da unidade 381201-4).

Estímulos discriminativos: seis formas não representativas, bidimensionais, pretas em fundo branco dentro de quadrados com bordas. As figuras foram arbitrariamente divididas em dois conjuntos de três (Conjunto 1: A1, B1 e C1; Conjunto 2: A2, B2 e C2; ver Figura 1). Os estímulos serão citados como positivos $(\mathrm{S}+)$ e negativos (S-), ao longo do texto. Sempre que os estímulos do Conjunto 1 eram positivos, os do Conjunto 2 eram negativos e vice-versa.

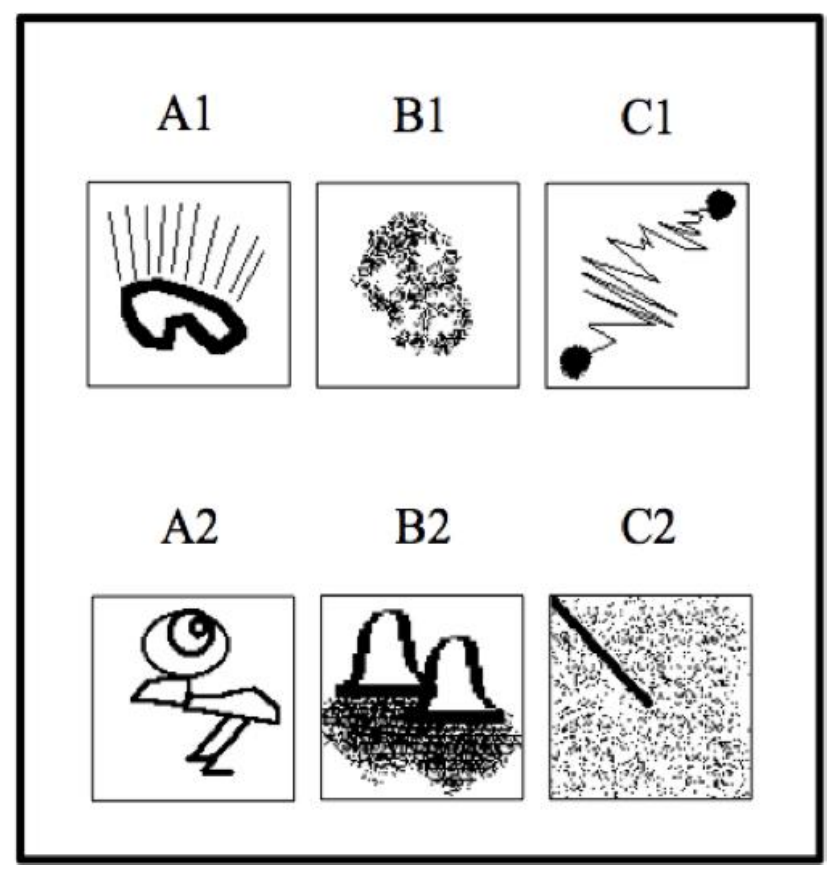

Figura 1. Estímulos utilizados no experimento. 
Estímulos reforçadores: pelotas de açúcar nos sabores framboesa e banana, conforme a disponibilidade.

Equipamento: um microcomputador Pentium Core 2 Duo (2,53-GHz), 2Gb (DDR2), executou o software EAM 4.0.04 (Desenvolvido por Dráusio Capobianco com financiamento do $\mathrm{CNPq}$ ) que controlou a apresentação das figuras (em nove possíveis posições de uma matriz $3 \times 3$, por meio de um monitor LCD de 17 " com tela sensível ao toque) e o registro das repostas de escolha do sujeito. O monitor foi acoplado a uma câmara experimental de acrílico e alumínio $(60 \times 60 \times 60 \mathrm{~cm})$ e ficava acessível ao sujeito por uma janela $(27 \times 33 \mathrm{~cm})$ em uma das paredes da câmara. Um dispensador de pelotas de $190 \mathrm{mg}$ foi utilizado para a apresentação das pelotas de comida. Um cronômetro foi utilizado para passagem de telas de time out e intervalo entre tentativas (ver procedimento).

Procedimento: $\mathrm{O}$ sujeito foi submetido a uma ou mais sessões experimentais diárias, cinco dias por semana (de segunda a sexta-feira em média, quatro horas antes da alimentação dos animais). Convencionou-se chamar aqui de sessão experimental um bloco de tentativas com número máximo definido e um critério de encerramento (descrito mais adiante no procedimento). Quando mais de uma sessão experimental era conduzida no mesmo dia, um intervalo de cinco a dez minutos decorria entre uma sessão e outra.

Todo o procedimento se baseou em treinos de discriminações simples, com estímulos funcionando como $\mathrm{S}+\mathrm{e}$ como S-. A cada tentativa, eram apresentadas de dois estímulos (no início do estudo) a seis (ao final), sendo metade dos estímulos negativos ( $\mathrm{S}$-) e a outra metade de estímulos positivos ( $\mathrm{S}+$ ). Os estímulos, qualquer que fosse a quantidade deles, eram apresentados simultaneamente no display (de fundo verde) do monitor. A resposta consistia em tocar duas vezes (razão fixa 2 - FR 2) qualquer ponto da área que um estímulo ocupava no display. Essa exigência foi progressivamente (de dois em dois) aumentada até oito (FR 8) para evitar respostas acidentais.

Quando mais de um S+ era apresentado, a tentativa era considerada concluída com acerto quando o sujeito escolhia apenas os $\mathrm{S}+$, em qualquer ordem. Assim, toques a um $\mathrm{S}+$ produziram sua remoção do display $\mathrm{e}$ a apresentação de uma pelota de comida, dando oportunidade para a próxima resposta a outro $\mathrm{S}+$. Quando apenas um S+ estava presente (seja porque era o único apresentado ou porque era o remanescente naquela tentativa), toques nele produziram a remoção de todos os estímulos do display, a apresentação de duas pelotas de comida e um intervalo entre tentativas (IET) de aproximadamente $6 \mathrm{~s}$. Uma das pelotas de comida era dispensada automaticamente no comedouro e a outra era apresentada pelo experimentador através do gradeado inferior da câmara e era considerada uma pelota-bônus, só apresentada quando o sujeito selecionava somente os $\mathrm{S}+$ na tentativa.

A qualquer momento de uma tentativa, toques ao estímulo arbitrariamente definido como $\mathrm{S}$ - produziram um time out inicialmente de $12 \mathrm{~s}$ (Fase 1), e posteriormente de 1 min (Fases 2 e 3), durante o qual a tela do computador ficava preta. O time out é uma consequência diferencial para erros que posterga a apresentação da próxima tentativa, portanto, atrasando a próxima oportunidade para obter comida. Ele tem o objetivo de tornar menos frequentes os erros. O time out era seguido pelo IET, sem apresentação de comida, e pela tentativa seguinte.

Desse modo, quando eram apresentados na tela do computador seis estímulos (três S+ e três S-), era possível ao sujeito apresentar até três respostas de escolha corretas, mas apenas uma errada, uma vez que uma primeira escolha errada encerrava a tentativa. Após o IET, seja após acerto ou erro, no início da tentativa seguinte, os estímulos eram apresentados em posições diferentes da tentativa anterior.

$\mathrm{O}$ procedimento incluiu um Pré-treino e três fases de Treino de Reversões, conforme descrito a seguir em ordem histórica. A diferença entre uma fase e outra consistiu no número de estímulos apresentados por tentativa (Pré-treino, dois estímulos; Fase 1, quatro estímulos; Fase 2, seis estímulos; Fase 3, ora quatro ora seis estímulos). O estudo envolveu outras variações no número de estímulos apresentados no início da tentativa, mas por serem alterações decorrentes diretamente da análise do desempenho do sujeito, serão especificadas e descritas apenas na seção de Resultados e Discussão.

Pré-treino, mudanças preparatórias. Discriminação simples simultânea com um $S+$ e um $S$ - (DS. 1.1). Foram apresentados seis pares de estímulos (um S+e um S-) com as seguintes contingências: 1) $\mathrm{A} 1+\mathrm{e} \mathrm{A} 2-$; 2) $\mathrm{A} 2+\mathrm{e} \quad \mathrm{B} 1-$; 3) $\mathrm{B} 1+\mathrm{e} \quad \mathrm{C} 2-$; 4$) \mathrm{C} 2+\mathrm{e} \quad \mathrm{C} 1-$; 5) $\mathrm{C} 1+\mathrm{B} 2-$; e 6) $\mathrm{B} 2+$ e A1-. Cada par era apresentado em uma sessão com no máximo 36 tentativas. $\mathrm{O}$ critério de precisão de desempenho para mudança de 
par era de seis acertos consecutivos (6ac). Alcançado esse critério, a sessão era encerrada mesmo que não tivessem sido apresentadas as 36 tentativas. Então, o par seguinte era apresentado até que o critério (6ac) fosse atingido, e assim sucessivamente até a apresentação do sexto par. O objetivo dessa etapa do procedimento foi avaliar o comportamento discriminativo dos sujeitos frente aos pares de estímulos, ao mesmo tempo em que cada um dos estímulos era apresentado tanto com função positiva quanto com função negativa. A Fase 1 foi iniciada após alcançado o critério de aprendizagem para a sexta discriminação.

Fase 1. Reversões de discriminações simples simultâneas com dois $S+e$ dois $S$ - (DS. 2.2). Tentativa iniciada com dois $\mathrm{S}+$ e dois $\mathrm{S}-(\mathrm{A} 1, \mathrm{~B} 1, \mathrm{~A} 2$ e B2). Neste tipo de tentativa, um acerto foi contado apenas quando a escolha dos dois estímulos positivos ocorreu, não importando a ordem. $\mathrm{O}$ critério de encerramento da sessão foi, inicialmente, de seis acertos consecutivos $(c-1=6 \mathrm{ac}$.). Uma análise do possível efeito desse critério sobre a precisão do desempenho do sujeito (ver seção de Resultados e Discussão) determinou uma mudança para um segundo critério $(c-2=$ encerramento 12 tentativas após $c-1$ ter sido atingido, independentemente do desempenho do sujeito nessas 12 tentativas adicionais). Um terceiro critério de precisão foi adotado no final da Fase $1(c-3=$ encerramento após a apresentação das 36 tentativas programadas, independentemente do número de acertos consecutivos contabilizados). Nesse caso, uma reversão poderia ou não ser feita na sessão seguinte a depender da análise da precisão do desempenho do sujeito nessas 36 tentativas. Essa análise era feita após a sessão e determinava o que seria feito na sessão seguinte. Sempre que o encerramento de uma sessão ocorria sem que o critério de seis acertos consecutivos tivesse sido alcançado, as contingências da sessão seguinte não eram revertidas. Havia, portanto, um único critério para processamento de uma reversão, que era o critério de seis acertos consecutivos.

Para efeito de descrição, a Fase 1 será então dividida em Subfases 1.1, 1.2, e 1.3, respectivamente de acordo com os critérios: $c-1, c-2$ e $c-3$.

Fase 2. Reversões de discriminações simples simultâneas com três $S+e$ três $S$ - (DS. 3.3). Tentativa iniciada com três S+ e três S- (A1, B1, C1, A2, B2 e C2). Neste tipo de tentativa um acerto foi contado quando o sujeito emitiu a resposta de escolha (oito toques) aos três estímulos positivos, não importando a ordem, sem que nenhuma resposta de escolha a Stivesse sido registrada. Não houve apresentação de pelota de comida a cada resposta de escolha, mas apenas ao final da tentativa, quando a mesma era concluída com acerto total. Isso tornou mais crítica a ocorrência de erros, uma vez que reforçamento só estava disponível após a conclusão de uma tentativa sem qualquer erro. Respostas a quaisquer dos estímulos negativos produziam inicialmente um time out de aproximadamente $12 \mathrm{~s}$. Com a obtenção dos primeiros resultados (ver Resultados e Discussão) a duração do time out foi aumentada para $1 \mathrm{~min}$ procurando garantir sua efetividade. O critério de encerramento da sessão após 36 tentativas (c-3 acima descrito) vigorou durante toda esta fase. A Fase 3 foi iniciada 10 reversões após o aumento na duração do time out.

Fase 3. Reversões parciais. Esta fase do experimento foi introduzida com o objetivo de avaliar o desempenho do sujeito quando uma parte dos estímulos tinha sua função revertida, enquanto outros estímulos eram omitidos. Assim, considerando as discriminações A1+/A2-, B1+/B2-, C1+/C2-, uma reversão era feita apenas com os estímulos A e B (A2+/A1-, $\mathrm{B} 2+/ \mathrm{B} 1-)$ ficando ausentes do treino de reversão os estímulos $\mathrm{C}$ ( $\mathrm{C} 1$ e $\mathrm{C} 2)$. Esse procedimento permite testar se as funções dos estímulos omitidos (Conjunto C) são espontaneamente revertidas. Se isso ocorre, é possível afirmar que os achados vão além do efeito de learning-set e podem ser considerados uma evidência de formação de classes funcionais (Vaughan, 1988).

Cada sessão de teste de reversão não treinada consistia de 36 tentativas. Nas 24 primeiras tentativas apenas quatro estímulos eram apresentados (dois do Conjunto 1, A1, B1 ou C1, e dois do Conjunto 2, A2, B2 ou C2 - Equivalente ao treino da Fase 1 - DS. 2.2). $\mathrm{O}$ critério de aprendizagem era de seis tentativas consecutivas concluídas com acerto (c-1). Caso esse critério fosse alcançado, os dois estímulos que tinham sido omitidos (um de cada conjunto) eram reintroduzidos nas 12 tentativas finais da sessão. As 12 tentativas finais eram idênticas às do treino da Fase 2 - DS. 3.3). Logo em seguida, uma sessão com 36 tentativas com seis estímulos era apresentada para avaliar o desempenho com os seis estímulos em uma sessão com o tamanho do treino padrão anterior.

$\mathrm{O}$ planejamento experimental definia que caso o critério $c-1$ não fosse alcançado nas 24 primeiras ten- 
tativas, as 12 tentativas restantes com seis estímulos não eram apresentadas. Uma sessão com 36 tentativas DS 3.3 era apresentada como retomada de linha de base antes que uma nova sessão de teste fosse apresentada. Esse procedimento permitiu avaliar a precisão de desempenho com um número menor de estímulos (quatro em vez de seis), bem como verificar o desempenho quando o par de estímulos omitidos foi reintroduzido no contexto experimental. A função dos estímulos apresentados nas 24 tentativas iniciais e nas 12 tentativas finais nas sessões de reversão parcial (parcial porque inicialmente somente quatro dos seis estímulos tinham sua função revertida) pode ser conferida na Tabela 1.

\section{Tabela 1}

Função e Forma de Apresentação dos Estímulos em Sessões de Reversão Parcial

\begin{tabular}{ccc}
\hline Reversão & 4 Estím. (DS. 2.2) & 6 Estím. (DS. 3.3) \\
\hline 1 & $\mathrm{~B} 1+\mathrm{C} 1+\mathrm{B} 2-\mathrm{C} 2-$ & $\mathrm{A} 1+\mathrm{B} 1+\mathrm{C} 1+\mathrm{A} 2-\mathrm{B} 2-\mathrm{C} 2-$ \\
2 & $\mathrm{~A} 1-\mathrm{C} 1-\mathrm{A} 2+\mathrm{C} 2+$ & $\mathrm{A} 1-\mathrm{B} 1-\mathrm{C} 1-\mathrm{A} 2+\mathrm{B} 2+\mathrm{C} 2+$ \\
3 & $\mathrm{~A} 1+\mathrm{B} 1+\mathrm{A} 2-\mathrm{B} 2-$ & $\mathrm{A} 1+\mathrm{B} 1+\mathrm{C} 1+\mathrm{A} 2-\mathrm{B} 2-\mathrm{C} 2-$ \\
4 & $\mathrm{~B} 1-\mathrm{C} 1-\mathrm{B} 2+\mathrm{C} 2+$ & $\mathrm{A} 1-\mathrm{B} 1-\mathrm{C} 1-\mathrm{A} 2+\mathrm{B} 2+\mathrm{C} 2+$ \\
5 & $\mathrm{~A} 1+\mathrm{C} 1+\mathrm{A} 2-\mathrm{C} 2-$ & $\mathrm{A} 1+\mathrm{B} 1+\mathrm{C} 1+\mathrm{A} 2-\mathrm{B} 2-\mathrm{C} 2-$ \\
6 & $\mathrm{~A} 1-\mathrm{B} 1-\mathrm{A} 2+\mathrm{B} 2+$ & $\mathrm{A} 1-\mathrm{B} 1-\mathrm{C} 1-\mathrm{A} 2+\mathrm{B} 2+\mathrm{C} 2+$
\end{tabular}

Nota. 4 Estím. (DS. 2.2) = os quatro estímulos apresentados durante as 24 tentativas primeiras tentativas; 6 Estím. (DS. 3.3) = Estímulos apresentados durante as 12 tentativas finais.

\section{RESULTADOS E DISCUSSÃO}

Foram efetuadas, ao todo, 112 sessões ao longo de nove meses. Foram realizadas cinco mudanças de funções discriminativas (Pré-treino) e 94 reversões das funções discriminativas dos estímulos (Fases 1 a 3), totalizando 3.918 tentativas. Devido a problemas com o controle experimental, foram realizadas seis reversões sem que o critério estabelecido tivesse sido alcançado (sinalizadas ao longo dos resultados). Essas seis reversões não foram consideradas nas análises subsequentes.

Pré-treino, mudanças preparatórias. Para todas as cinco mudanças, a precisão de desempenho estabelecida como critério foi atingida em, no máximo, 20 tentativas (Tabela 2, coluna "Num. de tent. até 6ac").

\section{Tabela 2}

Número de Tentativas Necessárias para Alcançar o Critério de 6ac nas Mudanças de Função Preparatórias

\begin{tabular}{ccc}
\hline Mudança & Contingência & Num. de tent. até 6ac \\
\hline 0 & A1+A2- & 7 \\
1 & A2+B1- & 18 \\
2 & B1+C2- & 9 \\
3 & C2+C1- & 13 \\
4 & C1+B2- & 9 \\
5 & B2+A1- & 20 \\
\hline
\end{tabular}


As discriminações adquiridas com número maior de tentativas ocorreram nas sessões em que um dos estímulos do Conjunto 2 era positivo (Mudanças 1, 3 e 5). Isto possivelmente ocorreu pelo fato de estas sessões terem sido antecedidas, no mesmo dia de coleta, por uma sessão com os estímulos do Conjunto 1 como positivos. Por se tratar de uma fase preparatória, na qual o objetivo era assegurar a emissão de um comportamento discriminado em relação aos estímulos, o desempenho foi considerado suficiente para o prosseguimento do experimento.

Fase 1. Reversões de discriminações simples simultâneas com dois $S+e$ dois $S$ - (DS. 2.2). Foram realizadas 64 reversões. O critério de encerramento $c$ 1 , encerramento imediato após 6ac, esteve em vigor nas 36 primeiras reversões. Quando mais de um estímulo positivo era apresentado, havia duas possibilidades de erros: 1) quando a primeira resposta de escolha era a um estímulo com função S- (denominado aqui erro Tipo 1), ou 2) quando a primeira resposta de escolha era a um estímulo positivo e depois ocorria uma resposta a um estímulo negativo (erro Tipo 2). Em ambos os casos era considerado que a tentativa não foi concluída com acerto. Durante o treino da primeira reversão verificou-se a persistência de erros Tipo 2. Procurando evitar esse problema, nas reversões 2 e 3 , foram apresentadas nove tentativas de discriminações simples com um $\mathrm{S}+\mathrm{e}$ dois $\mathrm{S}-(D S .1 .2)$ no início dessas sessões. O início deste tipo de tentativa era com um $\mathrm{S}+$ e dois $\mathrm{S}$-. A escolha de um $\mathrm{S}+$ produzia sua retirada, a apresentação de outro $S+$ em uma posição diferente do $\mathrm{S}+$ anterior e a liberação de uma pelota de comida. A escolha desse outro $\mathrm{S}+$ produzia a apresentação de uma pelota de comida, uma pelota bônus, o IET e a tentativa seguinte. A escolha de um S- produzia o encerramento da tentativa, o IET e a tentativa seguinte. Apenas um bloco deste tipo foi apresentado.

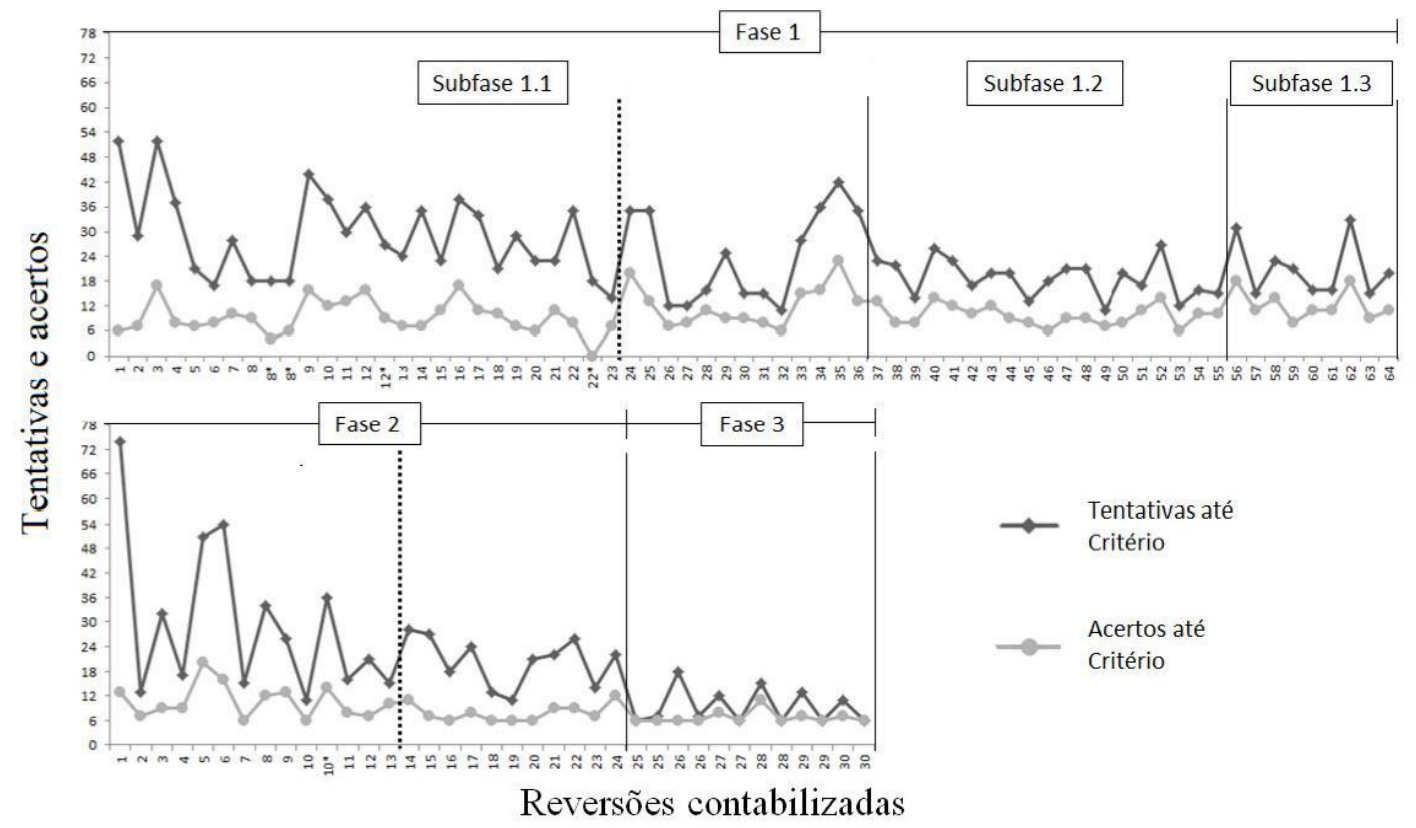

\begin{abstract}
Figura 2. Número de tentativas e de acertos até o critério de precisão definido (6ac) para cada reversão ao longo do estudo. 0 quadro superior corresponde às reversões da Fase 1 (Subfases 1.1, 1.2 e 1.3). A partir da linha tracejada na subfase 1.1 não houve mais apresentação de reforço para cada escolha correta, mas apenas ao final da tentativa. 0 quadro inferior corresponde às reversões da Fase 2 e Fase 3. A partir da linha tracejada o time out aumentou de 12-s para 1-min. Os asteriscos (no eixo x) indicam quando uma reversão foi repetida porque houve mudança nas contingências sem que 0 critério de precisão de desempenho tivesse sido alcançado. Nestes casos o número de tentativas e de acertos apresentados corresponde ao total daquela condição e não ao número até o critério definido.
\end{abstract}

Esta variação de procedimento (DS. 1.2 em blocos de nove tentativas) ocorreu diretamente em função dos resultados parciais obtidos e foi repetida na segunda reversão para evitar o mesmo tipo de erros na 
reversão seguinte. A partir de então, apenas tentativas DS. 2.2 foram utilizadas até a reversão número 23. A configuração do procedimento até este ponto do estudo, entretanto, não produziu reduções consistentes no número de tentativas (learning-set) e nem no número de acertos até o critério de 6ac (Figura 2, Fase 1, Sub-fase 1.1, reversões 1 a 23 ).

Com o objetivo de explorar possíveis melhorias no procedimento, considerando os resultados parciais obtidos até então, passou-se a não mais apresentar pelotas de comida a cada resposta de escolha correta, mas apenas ao final da tentativa, caso apenas os $\mathrm{S}+$ tivessem sido escolhidos. Esse procedimento foi adotado ao longo das reversões seguintes (24 a 64). A partir da reversão 24 até a 32 os dados mostraram alguma tendência de redução do número de tentativas requeridas até que o critério 6ac fosse atingido, embora certa variabilidade tenha sido observada em alguns pontos. Os dados obtidos nas reversões de 33 a 36, contudo, não mostram continuidade dessa tendência.

Esse mesmo tipo de instabilidade na precisão do desempenho foi observado em estudo anterior em nosso laboratório (De Man, 2007). Aparentemente os sujeitos aprendem que, com o critério de interrupção da sessão com seis tentativas corretas consecutivas, um desempenho muito preciso desde o início da sessão acaba por produzir perda de reforços com o encerramento precoce da sessão. Os dados obtidos por De Man (2007) e os dados obtidos aqui permitiram levantar a hipótese de que o critério de encerramento da sessão com seis tentativas corretas consecutivas $(c-1)$ pode selecionar um padrão de acertos e erros intercalados, elevando o número de tentativas processadas até que o referido critério seja atingido. A rigor, as contingências assim programadas poderiam selecionar como desempenho ótimo cinco acertos consecutivos e então um erro, de maneira que o máximo de tentativas seriam processadas, com o máximo de reforços possível sendo apresentados. Embora esse desempenho ótimo não tenha sido observado com grande frequência, o comportamento apresentado pelo sujeito parece se aproximar dele. Estudos posteriores deverão avaliar especificamente essa hipótese em procedimentos de treino discriminativo semelhante.

Essa análise levou à introdução das variações no encerramento das sessões (c-2 e $c$-3) com o objetivo de reduzir esses padrões de acertos e erros intercalados antes de 6ac. Com a adoção do critério $c$-2 (encer- ramento da sessão 12 tentativas após 6 ac [ver reversões 37 a 55 na Figura 2]), maior estabilidade no número de tentativas processadas até seis acertos consecutivos foi obtida. Não se observou, contudo tendência de redução do número de tentativas.

A adoção do critério $c$-3 (encerramento da sessão com 36 tentativas, [reversões 56 a 64]), não alterou a distribuição de acertos e erros. As variações de critérios $c-2$ e $c-3$ não afetaram o número de acertos até seis tentativas corretas consecutivas. O número de tentativas até 6ac nas Subfases 1.2 e 1.3 foi reduzido em relação ao momento anterior na Subfase 1.1, mantendo-se em torno de 24 tentativas (ver Figura 2).

Durante toda a Fase 1 não se observou uma curva descendente que indicasse um refinamento do desempenho, a despeito das variações no procedimento para encerramento de sessão. As variações no critério de encerramento das sessões $(c-2$ e $c-3)$ permitiram um número maior de tentativas após 6ac e, eventualmente, um número maior de acertos, mas não implicaram em uma curva de aprendizagem ao longo das reversões a cada mudança de critério. Como não houve maiores variações de desempenho após nove reversões desde o último ajuste de procedimento ( $c-3$, reversão 56, Figura 2), a Fase 2 foi iniciada.

Fase 2. Reversões de discriminações simples simultâneas com três $S+e$ três $S$ - (DS. 3.3). Foram realizadas 24 reversões. A Figura 2 apresenta o número de tentativas e a frequência de acertos até $6 \mathrm{ac}$ ao longo da Fase 2. No sentido de reduzir erros, e partir das variações de procedimento da fase anterior (DS. 1.2 , em blocos de nove tentativas no início das reversões), discriminações simples com um $S+e$ três $S$ (DS. 1.3) foram utilizadas somente para a inclusão dos estímulos C1 e C2 (Figura 2, Fase 2, Reversão 1) no treino com os outros quatros estímulos. Cada tentativa iniciou com um S+ e três S-. As escolhas corretas implicaram na retirada do estímulo positivo do display e apresentação de outro em posição diferente do S+ anterior. Da forma como foi apresentado, este tipo de tentativa apresentou um efeito transitório, isto é, houve aumento instantâneo da precisão do desempenho (Figura 2, Fase 2, Reversões 1 a 4), mas esse efeito não se manteve nas reversões seguintes (Figura 2, Fase 2, Reversões 5 e 6).

Em mais uma tentativa de aperfeiçoar o procedimento, o time-out foi aumentado de 12 para 60 segundos (Figura 2, Fase 2, linha vertical tracejada). Um 
aumento inicial no número de tentativas processadas até que seis tentativas corretas fossem atingidas foi observado (ver Reversões 14 e 15 na Fase 2 da Figura 2), mas uma tendência de queda a partir de então foi observada até a Reversão 19. Embora o número de tentativas processadas tenha ficando em torno de 24 tentativas, como na Fase 1, o desempenho na Fase 2 foi mais eficiente considerando que nesta fase havia um número maior de escolhas corretas em uma condição com um número maior de estímulos apresentados simultaneamente e consequentemente com maiores chances de erros.

Fase 3. Reversões parciais. Durante as 24 primeiras tentativas do procedimento de reversão parcial e durante as 12 tentativas com seis estímulos para toda a Fase 3, o desempenho mostrou-se ainda mais preciso e com maior tendência de queda do número de tentativas processadas até que seis tentativas corretas consecutivas fossem registradas (ver Figura 2, Fase 3, Reversões de 25 a 30). O desempenho nas 36 tentativas de retomada subsequente também foi mais preciso e mais estável. O número de tentativas e de acertos até 6ac foram também os menores documentados ao longo de todo o estudo.

Tomados juntos, os dados das Fases 1 e 2, e especialmente os dados da Fase 3, na Figura 2, é possível sugerir que o presente estudo documentou learning-set de reversões de discriminações simples combinadas. As variáveis dependentes que melhor evidenciam esse dado são o número de tentativa até $6 \mathrm{ac}$ (mostrando redução gradual nas Fases 2 e 3 ) e a frequência das sequências de escolha: uma análise minuciosa da frequência das sequências das respostas de escolha dos $\mathrm{S}+$ mostrou que o responder obtido não era controlado por um ou outro encadeamento de respostas corretas (isto é, não houve preferência por uma ou outra sequência).

Os procedimentos que mais parecem ter contribuído para essa demonstração foram: (1) reforçamento somente ao final de cada tentativa e não a cada escolha correta; (2) time out; e (3) reversões parciais. Em apenas seis reversões parciais foram documentados resultados superiores aos obtidos ao longo do extenso treino com apenas DS. 2.2 (64 reversões) ou apenas DS. 3.3 (24 reversões). O presente estudo encoraja estudos subsequentes sobre o efeito de learning-set de reversões de discriminações combinadas com o uso desses três procedimentos.
O procedimento adotado na Fase 3 permite, adicionalmente, avaliar se ocorreram reversões espontâneas, ou seja, se a experiência com a reversão das contingências com quatro estímulos, produziu reversão não treinada com o par remanescente dos estímulos ausentes até aquele momento. Esse seria um indício de formação de classes funcionais. Os dados obtidos nas seis reversões parciais (Fase 3), especialmente os dados das primeiras tentativas (dentre as 12, ver Tabela 3) quando o par remanescente é introduzido, permitem dizer que não foram encontradas evidências de formação de classes com a quantidade de reversões de discriminações processadas, embora a demonstração de desempenho preciso com o par remanescente a cada nova reversão tenha se tornado cada vez mais rápida. A Tabela 3 apresenta a ordem de escolha dos estímulos nas doze tentativas de reinserção de cada par omitido no treino inicial para cada uma das seis reversões parciais.

Os dados apresentados na Tabela 3 mostram que, embora o sujeito não tenha revertido prontamente a discriminação para o par omitido na primeira parte da reversão, a aquisição da discriminação com esse par foi rápida, de modo que, em todas as reversões, as três últimas tentativas da sessão foram concluídas com acerto e nas sessões com 36 tentativas DS. 3.3 apresentadas logo em seguida, o critério de 6 ac foi atingido no máximo de sete tentativas (Figura 2, Fase 3). Em outras palavras, a precisão do desempenho se tornou muito alta e foi crescente em apenas 12 tentativas.

Considerando as 72 tentativas processadas nessa situação de reinserção de pares de estímulo ausentes no início de uma reversão parcial (12 tentativas para cada uma das seis reversões parciais), e analisando a precisão do desempenho separadamente na primeira metade e na segunda metade desses blocos de 12 tentativas, é possível verificar que a precisão na primeira metade foi de 52,78\% (19 acertos em 36 tentativas) e na segunda metade foi de $88,89 \%$ (32 acertos em 36 tentativas).

Para uma análise ainda mais minuciosa, foi necessário diferenciar mais dois tipos de erros. Escolhas do estímulo negativo do par reinserido foram chamados de erros "X" e escolhas de estímulos negativos presentes nas 24 tentativas de reversão parcial foram chamados de erros "O". A Tabela 4 apresenta acertos (C) e erros ( $\mathrm{X} \mathrm{e} \mathrm{O}$ ) categorizados dessa forma. 
Tabela 3

Sequência de Estímulos Escolhidos (Por Exemplo, B1C1C2) nas Doze Tentativas em Que um Par de Estímulos Previamente Omitido (Reversão Parcial) foi Reinserido.

\begin{tabular}{ccccccc}
\hline & \multicolumn{7}{c}{ Reversões Parciais } \\
\cline { 2 - 7 } Tent & $\mathrm{A} 1+\mathrm{A} 2-$ & $\mathrm{B} 2+\mathrm{B} 1-$ & $\mathrm{C} 1+\mathrm{C} 2-$ & $\mathrm{A} 2+\mathrm{A} 1-$ & $\mathrm{B} 1+\mathrm{B} 2-$ & $\mathrm{C} 2+\mathrm{C} 1-$ \\
\hline 1 & $\mathrm{~B} 1 \mathrm{C} 1 \mathrm{C} 2$ & $\mathrm{C} 2 \mathrm{~A} 2 \mathrm{~B} 2$ & $\mathrm{~B} 1 \mathrm{~A} 1 \mathrm{C} 2$ & $\mathrm{C} 2 \mathrm{~B} 2 \mathrm{C} 1$ & $\mathrm{C} 1 \mathrm{~A} 1 \mathrm{C} 2$ & $\mathrm{~A} 2 \mathrm{~B} 2 \mathrm{C} 2$ \\
2 & $\mathrm{~B} 1 \mathrm{C} 1 \mathrm{C} 2$ & $\mathrm{~A} 2 \mathrm{C} 2 \mathrm{~B} 1$ & $\mathrm{C} 2$ & $\mathrm{~B} 2 \mathrm{C} 2 \mathrm{~A} 2$ & $\mathrm{C} 1 \mathrm{~A} 1 \mathrm{~A} 2$ & $\mathrm{C} 2 \mathrm{~A} 2 \mathrm{~B} 2$ \\
3 & $\mathrm{~B} 1 \mathrm{C} 1 \mathrm{~A} 2$ & $\mathrm{C} 2 \mathrm{~A} 2 \mathrm{~B} 2$ & $\mathrm{~B} 2$ & $\mathrm{~B} 2 \mathrm{C} 2 \mathrm{~A} 2$ & $\mathrm{~A} 1 \mathrm{C} 1 \mathrm{C} 2$ & $\mathrm{C} 2 \mathrm{~B} 2 \mathrm{C} 1$ \\
4 & $\mathrm{~B} 1 \mathrm{C} 1 \mathrm{~A} 1$ & $\mathrm{~B} 2 \mathrm{~A} 2 \mathrm{C} 2$ & $\mathrm{~A} 1 \mathrm{~B} 1 \mathrm{~A} 2$ & $\mathrm{~B} 2 \mathrm{C} 2 \mathrm{~A} 2$ & $\mathrm{~A} 1 \mathrm{C} 1 \mathrm{~A} 2$ & $\mathrm{~B} 2 \mathrm{C} 2 \mathrm{~A} 2$ \\
5 & $\mathrm{~B} 1 \mathrm{C} 1 \mathrm{~A} 2$ & $\mathrm{~B} 2 \mathrm{~A} 2 \mathrm{C} 2$ & $\mathrm{~A} 1 \mathrm{~B} 1 \mathrm{~B} 2$ & $\mathrm{~B} 2 \mathrm{C} 2 \mathrm{~A} 2$ & $\mathrm{~B} 1 \mathrm{C} 1 \mathrm{~A} 1$ & $\mathrm{C} 2 \mathrm{~A} 2 \mathrm{~B} 2$ \\
6 & $\mathrm{~B} 1 \mathrm{~A} 1 \mathrm{C} 1$ & $\mathrm{~B} 2 \mathrm{~A} 2 \mathrm{C} 2$ & $\mathrm{~B} 1 \mathrm{~A} 1 \mathrm{~A} 2$ & $\mathrm{~B} 2 \mathrm{C} 2 \mathrm{~A} 2$ & $\mathrm{C} 1 \mathrm{~A} 1 \mathrm{~B} 1$ & $\mathrm{~A} 2 \mathrm{~B} 2 \mathrm{C} 2$ \\
7 & $\mathrm{C} 1 \mathrm{~A} 1 \mathrm{~B} 1$ & $\mathrm{~B} 2 \mathrm{C} 2 \mathrm{~B} 1$ & $\mathrm{~B} 1 \mathrm{~A} 1 \mathrm{~B} 2$ & $\mathrm{C} 2 \mathrm{~B} 2 \mathrm{~A} 2$ & $\mathrm{C} 1 \mathrm{~B} 1 \mathrm{~A} 1$ & $\mathrm{C} 2 \mathrm{~B} 2 \mathrm{~A} 2$ \\
8 & $\mathrm{~B} 1 \mathrm{C} 1 \mathrm{~A} 1$ & $\mathrm{C} 2 \mathrm{~B} 2 \mathrm{~A} 2$ & $\mathrm{~A} 1 \mathrm{~B} 1 \mathrm{C} 1$ & $\mathrm{C} 2 \mathrm{~B} 2 \mathrm{~A} 2$ & $\mathrm{C} 1 \mathrm{~B} 1 \mathrm{~A} 1$ & $\mathrm{C} 2 \mathrm{~A} 2 \mathrm{~B} 2$ \\
9 & $\mathrm{C} 1 \mathrm{~B} 1 \mathrm{~A} 1$ & $\mathrm{~B} 2 \mathrm{C} 2 \mathrm{~A} 2$ & $\mathrm{~A} 1 \mathrm{~B} 1 \mathrm{~A} 2$ & $\mathrm{~B} 2 \mathrm{C} 2 \mathrm{~A} 2$ & $\mathrm{~A} 1 \mathrm{C} 1 \mathrm{~B} 1$ & $\mathrm{C} 2 \mathrm{~B} 2 \mathrm{~A} 1$ \\
\hline 10 & $\mathrm{~A} 1 \mathrm{~B} 1 \mathrm{C} 1$ & $\mathrm{C} 2 \mathrm{~B} 2 \mathrm{~A} 2$ & $\mathrm{~B} 1 \mathrm{~A} 1 \mathrm{C} 1$ & $\mathrm{C} 2 \mathrm{~A} 2 \mathrm{~B} 2$ & $\mathrm{~A} 1 \mathrm{C} 1 \mathrm{~B} 1$ & $\mathrm{C} 2 \mathrm{~B} 2 \mathrm{~A} 2$ \\
11 & $\mathrm{~B} 1 \mathrm{~A} 1 \mathrm{C} 1$ & $\mathrm{C} 2 \mathrm{~A} 2 \mathrm{~B} 2$ & $\mathrm{C} 1 \mathrm{~B} 1 \mathrm{~A} 1$ & $\mathrm{~B} 2 \mathrm{C} 2 \mathrm{~A} 2$ & $\mathrm{C} 1 \mathrm{~B} 1 \mathrm{~A} 1$ & $\mathrm{~B} 2 \mathrm{C} 2 \mathrm{~A} 2$ \\
12 & $\mathrm{~B} 1 \mathrm{~A} 1 \mathrm{C} 1$ & $\mathrm{~B} 2 \mathrm{~A} 2 \mathrm{C} 2$ & $\mathrm{C} 1 \mathrm{~B} 1 \mathrm{~A} 1$ & $\mathrm{C} 2 \mathrm{~B} 2 \mathrm{~A} 2$ & $\mathrm{~B} 1 \mathrm{C} 1 \mathrm{~A} 1$ & $\mathrm{C} 2 \mathrm{~B} 2 \mathrm{~A} 2$ \\
\hline
\end{tabular}

Nota. Os dados de cada reversão parcial são apresentados em dada uma das colunas. Os estímulos omitidos no treino durante as primeiras 24 tentativas de linha de base nomeiam as colunas. As células preenchidas em cinza indicam acertos completos na tentativa.

Organizados dessa forma, os dados possibilitam uma análise específica das respostas de escolha do sujeito ao par reinserido. Esse tipo de análise é importante para avaliar se alguma evidência de reversões espontâneas (e, portanto, de classes de estímulos) foi encontrada. A pergunta subjacente nessa análise é "o que o sujeito fez quando respondeu pela primeira vez aos estímulos reinseridos?" A ênfase na primeira ten- tativa é justificada por ser a única não afetada por reforçamento uma vez que ocorre antes das consequências programadas para acerto ou erro. A primeira resposta aos estímulos do par reinserido foi de acordo com as contingências revertidas (reversão espontânea) em quatro das seis reversões (Tentativa 1 da Reversão 2; Tentativa 2 da Reversão 4; Tentativa 5 da Reversão 5 e Tentativa 1 da Reversão 6).

Tabela 4

Acertos e Erros nas 12 Tentativas de Reinserção do Par não Revertido nas 24 Primeiras Tentativas de Cada uma das Seis Reversões Parciais

\begin{tabular}{|c|c|c|c|c|c|c|c|c|c|c|c|c|c|}
\hline \multirow{2}{*}{ Reversões } & \multirow{2}{*}{ Par reinserido } & \multicolumn{12}{|c|}{ Acertos (C), Erros (X e O) por tentativa (1 a 12) } \\
\hline & & 1 & 2 & 3 & 4 & 5 & 6 & 7 & 8 & 9 & 10 & 11 & 12 \\
\hline 1 & $\mathrm{~A} 1+\mathrm{A} 2-$ & 0 & 0 & $X$ & C & $x$ & $C$ & C & C & C & C & C & C \\
\hline 2 & $\mathrm{~B} 2+\mathrm{B} 1-$ & C & $X$ & C & C & C & C & $x$ & C & C & C & C & C \\
\hline 3 & $\mathrm{C} 1+\mathrm{C} 2-$ & $\mathrm{X}$ & $\mathrm{X}$ & 0 & 0 & 0 & 0 & 0 & C & 0 & C & C & C \\
\hline 4 & $A 2+A 1-$ & 0 & C & C & C & C & C & C & C & C & C & C & $C$ \\
\hline 5 & $\mathrm{~B} 1+\mathrm{B} 2-$ & 0 & 0 & 0 & 0 & C & C & C & C & C & C & C & C \\
\hline 6 & $\mathrm{C}_{2}+\mathrm{C} 1-$ & C & C & $x$ & C & C & C & C & C & 0 & C & C & C \\
\hline
\end{tabular}

Embora se possa dizer que quatro respostas corretas em um total de seis ocasiões sejam ainda evidência fraca de reversão espontânea das discriminações com os estímulos omitidos na fase de reversão parcial, esses dados indicam que as alterações de procedimento aqui processadas geraram um procedimento final 
promissor para o estudo de learning-set de reversões de discriminações e formação de classes funcionais.

Os dados das Reversões 2, 4, 5 e 6 são especialmente animadores porque mostram acerto na primeira vez que o sujeito respondeu a estímulos do par remanescente e quase nenhum erro subsequente. Esses dados sugerem que, não apenas learning-set de reversões foi encontrado, mas que também, em um estudo subsequente, possivelmente um número maior de reversões parciais poderá levar a documentar evidências convincentes de reversão espontânea e, consequentemente, de formação de classes.

O presente trabalho apresenta a concepção de um procedimento de treino de repetidas reversões de discriminações simples combinadas com seis estímulos. Esse arranjo experimental permite a busca por evidências de learning-set de reversões e incorpora o procedimento de reversões parciais (Dube, Callahan \& Mcllvane, 1993; Dube, McIlvane, Callahan \& Stoddard, 1993; Reis \& Costa, 2002). Além disso, esse procedimento apresentou a vantagem de evitar o desenvolvimento de relações de controle não programadas, frequentemente observado em treino extensivo de reversões de discriminações simples com duas escolhas (Beran et al., 2008), e não produziu mero controle por sequências de respostas ao longo dos treinos de reversões de discriminações.

Comparado ao estudo de Vaughan (1988), o presente estudo aponta um procedimento que preserva algumas das características daquele estudo pioneiro, mas se mostra mais exequível pela quantidade menor de reversões necessárias. Esse avanço segue a mesma linha de busca por procedimentos mais eficazes para estudar learning-set de reversões já iniciada por Costa (2008), Costa et al. (2007) e Dahás, Brasiliense, Barros, Costa e Souza (2010).

A partir das variações de critério de encerramento realizadas, parece seguro afirmar que encerramentos de sessões com poucas tentativas após o critério ( $\mathrm{Fa}$ se 2, critério $c-1$ ), ou mesmo muitas tentativas, mas com consequência diferencial não significativa para erros (Fase 2, critérios c-2 e c-3, time out 12-s), foram aspectos que produziram erros antes de $6 \mathrm{ac}$ com maior frequência. Esses resultados corroboram as evidências de De Man (2007), quando discute a origem do que se chama de "escores intermediários" na aprendizagem de discriminações simples (De Man, 2007). A utilização de um time out relativamente longo (1-min) com a permissão de mais tentativas após 6ac reduziu a frequência de acertos intercalados antes do critério. Estudos posteriores podem avaliar a generalidade desses dados para outras condições experimentais, outros sujeitos e tarefas.

Goulart, Galvão e Barros (2003, Experimento 2, com três pares de estímulos), descreveram resultados com variações nas porcentagens de acerto semelhantes às descritas no presente estudo e também enfrentaram dificuldades para o estabelecimento da precisão de desempenho planejada para prosseguimento entre fases. Ao repensarem o procedimento (Experimento 3, agora utilizando dois pares de estímulos somente), observaram um aumento considerável das porcentagens de acerto em relação à tarefa com seis estímulos por sessão. O presente estudo relata evidências correlatas a esses resultados, na medida em que as reversões parciais (com quatro estímulos) foram efetuadas sistematicamente em um menor número de tentativas e com índices de acerto globais maiores do que todos os registrados até aquele momento. Os desempenhos para os dois tipos de tarefas durante as reversões parciais foram similares e melhores para a tarefa com seis estímulos, se considerados em valores absolutos. Isto sugere que apresentar uma tarefa mais simples, com um menor número de elementos, após uma tarefa mais complexa, com um maior número de elementos, parece favorecer o controle de estímulos relevante. Essa questão sobre a organização do treino, assim como aquelas relativas ao efeito dos procedimentos de reforçamento somente ao final de cada tentativa (e não a cada escolha correta) de uso de time out de 1 min. e de reversões parciais, são aspectos que devem ser aprofundados em novos estudos que busquem avaliar o papel do learning-set em tarefas de reversões repetidas como um preditor da formação de classes funcionais.

\section{REFERÊNCIAS}

Beran, M. J., Klein, E. D., Evans, T. A., Chan, B., Flemming, T. M., Harris, E. H., Washburn, D. A., \& Rumbaugh, D. M. (2008). Discrimination reversal learning in capuchin monkeys (Cebus apella). The Psychological Record, 58, 3-14.

Costa, T. D., (2008). Repetidas reversões de discriminações simples e formação de classes funcionais em animais (Tese de doutorado). Universidade Federal do Pará, Belém. Retirado de http://www.ufpa.br/ppgtpc/dmdocuments/DOUTORADO/TES EThiagoCosta2008.pdf

Costa, T. D., Barros, R. S., Galvão, O. F., \& Reis, M. J. D. (2007). Aquisição de repertório discriminativo auditivo em esquema múltiplo: Explorando procedimento para estudo da formação 
de classes funcionais em ratos. Revista Brasileira de Análise do Comportamento, 3, 65-77.

Dahás, L., Brasiliense, I., Barros, R. S., Costa, T. D., \& Souza, C. B. A. (2010). Formação de classes funcionais em cães domésticos (Canis familiaris): Uma abordagem da aquisição de comportamento pré-simbólico. Acta Comportamentalia, 18, 317346.

De Man, T. S. L. (2007). Efeito do treino de discriminações simples sobre o repertório de pareamento ao modelo por identidade de um macaco-prego (Cebus apella) (Dissertação de mestrado). Universidade Federal do Pará, Belém. Retirado de http://www.ufpa.br/ppgtpc/dmdocuments/MESTRADO/Tiagod eMan2007.pdf

Dube, W. V., Callahan, T. D., \& McIlvane, W. J. (1993). Serial reversals of concurrent auditory discriminations in rats. The Psychological Record, 43, 429-440.

Dube, W. V., McIlvane, W. J., Callahan, T. D., \& Stoddard, L. T. (1993). The search for stimulus equivalence in nonverbal organisms. The Psychological Record, 42, 761-778.

Goulart, P. R. K., Galvão, O. F., \& Barros, R. S. (2003). Busca de formação de classes de estímulos via procedimento de reversões repetidas de discriminações simples em macaco-prego (Cebus apella). Interação em Psicologia, 7, 109-119.
Harlow, H. F. (1949). The formation of learning sets. Psychological Review, 56, 51-65.

Kastak, C. R., Schusterman, R. J., \& Kastak, D. (2001). Equivalence classification by California sea lions using class-specific reinforcers. Journal of the Experimental Analysis of Behavior, 76, 131-158.

Lionello-DeNolf, K. M., McIlvane, W. J., Canovas, D., de Souza, D., \& Barros, R. S. (2008). Reversal learning set and contingency class formation in children with and without autism. The Psychological Record, 58, 15-36.

Reis, M. J. D., \& Costa, T. D. (2002). Discriminação concorrente em sessões de longa duração: Uma contribuição metodológica. Arquivos Brasileiros de Psicologia, 54, 304-317.

Vaughan, W. (1988). Formation of equivalence sets in pigeons. Journal of Experimental Psychology: Animal Behavior Processes, 14, 36-42. 